Turkish Journal of Geriatrics

DOI: 10.31086/tigeri.2020.152

2020; 23(2): 180-187

- Cigdem AKYOL BEYOGLU 1 (D)

- Serkan TEKSOZ² D

CORRESPONDANCE

Cigdem AKYOL BEYOĞLU

Istanbul University, Cerrahpasa, Cerrahpasa

School of Medicine , Department of

Anesthesiology and Reanimation, Istanbul,

TURKEY

Phone: +905052278176

e-mail: akyolbeyoglu@gmail.com

Received: January 24, 2020

Accepted: April 20, 2020

Istanbul University- Cerrahpasa, Cerrahpasa

School of Medicine, Department of

Anesthesiology and Reanimation, Istanbul,

TURKEY

${ }^{2}$ Istanbul University- Cerrahpasa, Cerrahpasa School of Medicine, Department of General Surgery, Istanbul, TURKEY

\section{INVESTIGATION OF THE EFFECT OF PREOPERATIVE DEMOGRAPHIC CHARACTERISTICS ON RESPIRATORY AND CARDIOVASCULAR COMPLICATIONS AFTER A THYROIDECTOMY IN GERIATRIC PATIENTS; A RETROSPECTIVE STUDY}

\section{Abstract}

Introduction: Recently, patients are discharged home between 6 to 24 hours after thyroidectomy. The safety of this method in geriatric patients is not clear. Our aim is to investigate the effect of preoperative demographic characteristics on respiratory and cardiovascular complications after total thyroidectomy in geriatric patients who are over 65 years.

Materials and Method: After obtaining local ethical committee approval, the perioperative data of geriatric patients who had total thyroidectomy between January 2016 and December 2018 were evaluated. Patients' age, sex, body mass index, American Society of Anesthesiologists score, preoperative systemic disease or medical comorbidities such as thyroid hormone dysfunction and electrolyte imbalance, difficult airway, operation time, preoperative vocal cord paralysis and thyroid malignancy were evaluated. Patients with respiratory or cardiovascular complications 30 days after surgery were evaluated about the effect of demographic data by using one -way ANOVA test.

Results: Of the 191 patients in this retrospective analysis, 10 of the patients suffered from respiratory complications and 13 suffered cardiovascular complications. Patients who were observed respiratory complications had a higher body mass index compared to others $(p=0.043)$. Patients with cardiovascular complications had an older age and higher systolic and diastolic blood pressure prior to surgery $(p=0.013, p<0.01, p=0.032$, respectively). Systemic diseases and medical comorbidities were not associated with postoperative complications.

Conclusions: Systemic diseases and medical comorbidities may not increase postoperative complication risk in geriatric patients undergoing outpatient total thyroidectomy. Postoperative complications in these patients may be related to respiratory or cardiovascular risk factors prior to surgery.

Key words: Aged; Surgery; Comorbidity; Thyroidectomy. 


\section{INTRODUCTION}

The aging population has increased in parallel with technological developments and advances in medical care. The total thyroidectomy rate due to thyroid diseases has increased in the geriatric population. In recent years, patients, even geriatric patients are discharged within 6-24 hours postoperatively following a total thyroidectomy to ensure that medical interventions are performed in the most cost-effective manner (1).

The World Health Organization defines a chronological age of 65 years as elderly (2).

Postoperative morbidity and mortality rates are high in geriatric patients due to cardiovascular diseases, drugs used due to treat concomitant diseases, and physiological problems caused by advanced age (3). To address this issue, factors linked to postoperative complications in the geriatric population should be investigated to identify which patients are at risk of complications after early discharge.

The main complications of thyroid surgery are laryngeal nerve injury, hypocalcemia and bleeding (4). Given the comorbidities that often accompany advanced age, surgical procedures may be risky in this population (5). Thus the treatment of thyroid diseases in the elderly requires special attention due to the increased risk of complications.

Previous studies on the effect of age on postthyroidectomy complications failed to show a clear safety risk of surgery among geriatric patients $(1,2,6)$. However, there is a lack of evidence about how ageing effects when there is a medical comorbidity such as hypertension or electrolyte imbalance.

The aim of this study was to determine the risk factors related to postoperative respiratory or cardiovascular complications in patients older than 65 years of age with a diffuse goiter or malignancy who underwent a total thyroidectomy in the general surgery operating room of Istanbul University- Cerrahpasa, Cerrahpasa School of
Medicine.

\section{MATERIALS AND METHODS}

After obtaining the approval of the local ethics committee, we examined the preoperative anesthesia evaluation forms, perioperative anesthesia-recovery room follow-up slips and surgical records of all patients aged older than 65 y who underwent a total thyroidectomy performed between January 2016 and December 2018 in Istanbul University- Cerrahpasa, Cerrahpasa School of Medicine General Surgery Operating Room.

Each patient's age, sex, height, weight, body mass index (BMI), American Society of Anesthesiologists (ASA) score, preoperative comorbidities (hypertension, heart disease, asthma, chronic obstructive pulmonary disease, thyroid hormone dysfunction, and electrolyte imbalance) were recorded, in addition to airway related factors (i.e., a difficult airway), operation time, preoperative vocal cord paralysis and reason for the surgery (i.e., presence or absence of malignancy).

Thyroid hormone dysfunction (THD) was considered as any deviation from the reference values (thyroid stimulating hormone [TSH]: 0.46 - $4.68 \mathrm{mU} / \mathrm{L}$, T4: 0.7-2.0 ng/dL). THD patients in whom oral medications failed to normalize $\mathrm{TSH}$ and T4 values underwent surgery. Huge deviations from normal ranges were treated with plasmapheresis.

Any respiratory or cardiovascular complications $30 \mathrm{~d}$ after surgery were recorded.

Respiratory complications included the following: PO2 desaturation of $<90 \%$ under 4 $\mathrm{L} / \mathrm{min} \mathrm{O} 2$ via a face mask in the recovery room, dyspnea, respiratory insufficiency, pneumonia, pulmonary embolism, and ventilator requirement for $>48$ h. Cardiovascular complications included: new-onset arrhythmias, myocardial infractions, and cerebrovascular events. The same surgeon (S.T.) performed all the thyroidectomies. An 
endotracheal monitoring system (Medtronic NIM, Jacksonville, FL) was used to monitor neural stimulation with $1.5 \mathrm{~mA}$.

In this study, which was conducted to investigate the relationship between demographic data and respiratory and cardiovascular complications after a total thyroidectomy in the geriatric patient population, the power (test power) of each variable was determined to be at least 0.80 and Type 1 Error 0.05. The number of sampling was determined to be 190 by the single-stage random probability sampling method based on the main population ratios. Descriptive statistics were applied to continuous variables with the data presented as the mean, standard deviation, minimum and maximum. Categorical variables were expressed as numbers and percentages. Kolmogorov-Smirnov ( $\mathrm{n}>50)$ and SkewnessKurtosis tests were conducted to determine whether the data were normally distributed. Parametric tests were performed due to a normal distribution. An independent T-test or a one-way analysis of variance was used to compare the mean values of postoperative complication groups. A chi-square test was used to determine the relationship between postoperative complications and other categorical variables. The statistical significance level $(\alpha)$ was taken as $5 \%$ in the calculations. The SPSS (IBM SPSS for Windows, ver.24; IBM) statistical package program was used for all calculations.

\section{RESULTS}

In total, 191 patients (females, $n=118$; males, $n=73$ ) older than 65 y were included in this retrospective study between January 2016 and December 2018. The mean age of the patients was $69.27 \mathrm{y}$, the mean height was $162.16 \mathrm{~cm}$, and the mean weight was $76.26 \mathrm{~kg}$. The mean BMI was $27 \mathrm{~kg} / \mathrm{m} 2$, and the mean ASA score was 2.05. The demographic data are given in Table 1. Respiratory complications were observed in 10 patients (5.2\%), cardiovascular complications were observed in 13 patients (6.8\%).

The mean BMI of the patients with respiratory complications was higher than that of the other patients $(p=0.043)$ (Table 1). The mean age of patients with cardiovascular complications was also higher than that of the other patients $(p=0.013)$ (Table 1). The mean systolic and diastolic arterial pressure values of patients with cardiovascular complications were higher than those of patients without complications $(p<0.01$ and $p=0.032$, respectively) (Table 2).

The duration of the operation, postoperative NRS scores, and pre-postoperative vocal cord paralysis were similar among the patients (Table 2).

Three of 10 (\%23.1) patients who experienced cardiovascular complications and 37 of 168 (22\%) patients without any complications had THD before the operation. Two of 10 (20\%) patients who experienced respiratory complications and 16 of 168 (9.5\%) patients who did not experience any complications had an electrolyte imbalance prior to surgery. Neither THD nor an electrolyte imbalance had an effect on postoperative complications $(p=0.24$ and $p=0.26$, respectively) (Table 2).

Six of 10 patients (60\%) who experienced cardiovascular complications were hypertensive, and one patient had ischemic heart disease. Stridor was observed in 8 of 10 (80\%) patients who developed respiratory complications. Stridor was not observed in any of the patients who developed cardiovascular complications and only in $1(0.1 \%)$ patient who was not observed any complication $(p<0.01)$.

One patient had a difficult airway and had a narrow airway passage in the glottic area according to the ear nose throat surgeon consultation. This patient had a Mallampati score of 4 and was intubated using a video laryngoscope (C-MAC $\mathbb{R}$ $S$ video laryngoscopes, Storz, Germany) with an obturator used in the second attempt. The patient had no adverse respiratory events after extubation.

None of the patients were referred to the post anesthesia care unit after surgery.

All the patients were discharged within $23 \mathrm{~h}$ after surgery. 
Table 1. Demographic data.

\begin{tabular}{|l|r|r|r|r|}
\hline & RC & CVC & None \\
\hline Number of patients & 10 & 13 & 168 & \\
\hline Mean age (years) & 67 & $72^{\star}$ & $101(60.1)$ & $<0.01$ \\
\hline Female sex [n(\%)] & $6(60)$ & $11(84.6)$ & 29 & 0.21 \\
\hline BMI (kg/m2) & $33^{\star}$ & 29 & 2.07 & 0.04 \\
\hline ASA classification & 2.4 & 2.07 & 2.03 \\
\hline
\end{tabular}

*statistically significant compared to the other groups. RC: Respiratory complication group. CVC: Cardiovascular complication group BMI: Body mass index. ASA: American Society of Anesthesiologists. None: Patients without any complications

Table 2. Perioperative data and medical conditions of all patients.

\begin{tabular}{|c|c|c|c|c|}
\hline & RC & CVC & None & p \\
\hline $\mathrm{OD}(\min )$ & 56 & 50 & 54 & 0.61 \\
\hline $\mathrm{SAP}(\mathrm{mm} \mathrm{Hg})$ & 135 & $166^{\star}$ & 143 & $<0.01$ \\
\hline $\mathrm{DAP}(\mathrm{mm} \mathrm{Hg})$ & 77 & $89 *$ & 82 & 0.03 \\
\hline NRS & 2.60 & 2.23 & 2.39 & 0.69 \\
\hline Malignancy[n(\%)] & $1(10)$ & $2(15)$ & $24(14.2)$ & 0.38 \\
\hline NVS $>1[n(\%)]$ & $2(20)$ * & $1(7.7)$ * & $3(1.8)$ & 0.04 \\
\hline $\mathrm{THD}[\mathrm{n}(\%)]$ & $0(0)$ & $3(23.1)$ & $37(22)$ & 0.24 \\
\hline $\mathrm{El}[\mathrm{n}(\%)]$ & $2(20)$ & $0(0)$ & $16(9.5)$ & 0.26 \\
\hline Pre VCP[n(\%)] & $2(20)$ & $0(0)$ & $3(1.8)$ & 0.08 \\
\hline Post VCV[n(\%)] & $2(20)$ & $0(0)$ & $11(6.6)$ & 0.2 \\
\hline
\end{tabular}

*: Statistically significant. OD: Operation duration. SAP: Systolic arterial pressure. DAP: Diastolic arterial pressure. NRS: Numeric rating scale score. NVS: Nausea and vomiting scale score. THD: Thyroid hormone dysfunction. El: Electrolyte imbalance. Pre VCP: Preoperative vocal cord paralysis (Right or left) Post VCV: Postoperative vocal cord paralysis (Right or left). None: Patients without any complications. 


\section{DISCUSSION}

Thyroid diseases are common among elderly patients (2). Although there are concerns about the negative effects of aging on mortality and morbidity after thyroid surgery, this issue has not been clarified previously. In recent years, the majority of patients who have undergone thyroid surgery are discharged within 24 h. $(1,6)$. However, comorbidities in geriatric patients may give rise to complications after thyroid surgery. In the current study, older age and hypertension were risk factors for postoperative cardiovascular complications, and a higher BMI was a risk factor for the development of postoperative respiratory complications.

In previous studies $(3,7,8)$ older age (i.e., $>70$ y) was a risk factor for postoperative complications after outpatient thyroidectomy. The results of these studies are in accordance with those of the present study. In contrast, one previous study found that a thyroidectomy could be safely performed in elderly and very elderly patients (1). However, this study focused on different postoperative surgical complications, particularly the development of hypocalcemia, postoperative hematomas, and recurrent laryngeal nerve injury. A number of studies suggested that comorbidities in the elderly population seemed to be responsible for postoperative complications $(9,10)$. In the present study, 7 of 10 patients who had cardiovascular complications postoperatively experienced cardiovascular diseases (hypertension and ischemic heart disease).

As reported previously, the incidence of heart diseases increases with ageing $(11,12)$. The mean systolic and diastolic arterial pressure values in patients with cardiovascular complications were higher than those in patients without complications. We are not surprised about the cardiovascular effects of patients with heart diseases in our study. Based on the results, we conclude that patients with cardiovascular diseases preoperatively had cardiovascular complications postoperatively. However additional medical comorbidities such as THD, or El were not related to cardiovascular complications.

In the previous study, patients with respiratory complications had a higher BMI than that of the other patients. Previous studies demonstrated an association between morbid obesity and postoperative respiratory complications in various types of surgery $(13,14,15)$. Research also reported that obesity was an important risk factor for the development of asthma, obstructive sleep apnea, obesity hypoventilation syndrome, and pulmonary hypertension (16). In the same study, chest wall and respiratory system compliance were decreased in morbidly obese patients, and morbidly obese patients were prone to atelectasis and respiratory insufficiency (16). Furthermore, obese geriatric patients were shown to have severe skeletal muscle dysfunction, which reduced their quality of life and survival (17). In the present study, the mean $\mathrm{BMI}$ of the patients with respiratory complications was $33 \mathrm{~kg} / \mathrm{m} 2$. This result is in accordance with that of previous studies $(16,17,18,19)$. In contrast, preoperative unilateral vocal cord paralysis had no effect on postoperative respiratory complications in the present study. As surgeons in our hospital use a neural monitoring system to ensure nerve integrity, the contralateral recurrent nerve was not damaged in any of the patients. No respiratory complications were observed in patients who had unilateral vocal cord paralysis before surgery.

Stridor is an abnormal, high-pitched sound produced by turbulent airflow through a partially obstructed airway at the level of the supraglottis. In the present study, stridor was observed in 8 of 10 patients who developed respiratory complications in our study. Stridor after a thyroidectomy may indicate a laryngeal edema, laryngeal nerve palsy or laryngeal dysfunction secondary to hypocalcemia. Seven of the study patients with stridor had a BMl greater than $30 \mathrm{~kg} / \mathrm{m} 2$. As since stridor was transient and treated in the recovery room, we believe stridor observed in our patients was related to laryngeal edema. In cases of stridor observed in the recovery room after a 
thyroidectomy, anesthesiologists must be alert to the possibility of laryngeal edema, laryngeal nerve palsy or acute hypocalcemia.

The results of the previous study revealed no association between medical comorbidities and postoperative complications. According to our results, neither preoperative thyroid hormone dysfunction nor an electrolyte imbalance affected postoperative complications.

Hyperthyroidism has profound effects on the heart. These effects include tachycardia, arrhythmias, increased systolic pressure, increased systolic function, left ventricular hypertrophy, and diastolic dysfunction. However an adaptation mechanism may restore patients' health status and hyperthyroidic patients may not be adversely affected in cases of a thyroid hormone imbalance $(18,20)$. In common with the findings of the present study, Pitzalis et al (21) found no correlation between hyperthyroidism and increased cardiac anomalies. We think this issue needs to be further evaluated. During anesthesia and surgery, hypothyroid patients have an elevated risk of complications including heart conduction anomalies, hemostasis problems and decreased pulmonary ventilation. We expected that a combination of older ages and hypothyroidism would have adverse effects on cardiovascular and respiratory functions. However in accordance with the findings of a previous study (19), hypothyroidism was not associated with postoperative cardiovascular and respiratory complications. In the previous study, anemia and hypertension were more prevalent in hypothyroid patients, but this was not related to adverse events.

Although serum TSH levels increase slightly with age, cardiovascular adverse events related to hypothyroidism are uncommon in the elderly (22). Furthermore, hypothyroidism and aging have similar effects on the basal metabolic rate, thereby raising the question whether hypothyroidism is a protective adaptive mechanism for the aging body. Previous studies demonstrated that hypothyroidism was shown to deteriorate the existing cardiac disease in the elderly. However, in the present study, unattended hypothyroidism was not related to adverse cardiovascular events, similar to that found in previous studies $(22,23)$

In addition to THD, profound hypo- and hypercalcemia were not seen in any of our patients perioperatively. A mild electrolyte imbalance was not associated with adverse events in our study, similar to that found in previous research (3).

Some authors found a relation between malignancy and postoperative complications rates after a thyroidectomy $(3,24)$. However, these complications involved surgery- related complications such as a subcutaneous hematoma, hypocalcemia, or recurrent nerve palsy. The extent of surgery, invasion of the surrounding tissues, and preoperative recurrent nerve palsy related to thyroid malignancy may result in increased surgical complications. Other studies reported contrary results, like our study $(1,25)$. To date, it has not been shown to be related to respiratory and cardiovascular complications. This finding is in accordance with our results.

In conclusion, there are conflicting results on the safety of elderly outpatient thyroid surgery. The results of our study may confirm that patients with respiratory or cardiovascular risk factors prior to surgery are at risk of postoperative complications, whereas medical comorbidities such as THD, electrolyte imbalances, neoplasms and older age are not additional risks causing postoperative complications. We believe geriatric patients may safely undergo thyroid surgery, even in the presence of additional medical comorbidities, when prepared meticulously for the surgery and anesthesia. In common with the adult population, geriatric patients may undergo outpatient thyroidectomy, without additional risks.

\section{Acknowledgement}

None

\section{Conflict of interest}

Authors declare no conflict of interest 


\section{REFERENCES}

1. Grubey JS, Raji Y, Duke WS, Terris DJ. Outpatient thyroidectomy is safe in the elderly and super-elderly. Laryngoscope. 2018;128(1):290-4. (PMID: 28573781).

2. Teksöz S, Arikan AE, Ferahman S, et al. Effect of total thyroidectomy on mortality and morbidity in geriatric patients: Cerrahpasa experience. Turkish Journal of Geriatrics. 2013;16(4):365-71.

3. Caulley L, Johnson-Obaseki $S$, Luo L, Javidnia H. Risk factors for postoperative complications in total thyroidectomy: A retrospective, risk-adjusted analysis from the National Surgical Quality Improvement Program. Medicine (Baltimore). 2017;96(5):e-5752. (PMID: 28151852).

4. Assenza M, Ricci G, Romagnoli F, Binda B, Rengo M. Thyroid surgery: total and partial resection. Analysis of complications and a review of the literature. Chir Ital. 2004;56(3):371-82. (PMID: 15287634).

5. Chiovato L, Mariotti S, Pinchera A. Thyroid diseases in the elderly. Baillieres Clin Endocrinol Metab. 1997;11(2):251-70.(PMID: 30606015).

6. Meltzer C, Clau M, Gurushanthaiah D, Di Meng, Linda Radler AS. Safety of Outpatient Thyroid and Parathyroid Surgery: A Propensity Score-Matched Study. Otolaryngol - Head Neck Surg (United States). 2016;154(5):789-96.(PMID: 27006296).

7. Khavanin N, Mlodinow A, Kim JYS, Ver Halen JP, Antony AK, Samant S. Assessing Safety and Outcomes in Outpatient versus Inpatient Thyroidectomy using the NSOIP: A Propensity Score Matched Analysis of 16,370 Patients. Ann Surg Oncol. 2015;22(2):429-36. (PMID: 24841353).

8. Bliss R, Patel N, Guinea A, Reeve TS, Delbridge L. Age is no contraindication to thyroid surgery. Age Ageing. 1999;28(4):363-6.(PMID 10459789).

9. Fallahzadeh H, Mays ET. Preexisting disease as a predictor of the outcome of colectomy. Am J Surg. 1991;162(5):497-8.(PMID 1951920).

10. Simmonds PD, Best $L$, George $S$, et al. Surgery for colorectal cancer in elderly patients: A systematic review. Lancet. 2000;356(9234):968-74.(PMID: 11041397).

11. Singh $M$, Stewart $R$, White $H$. Importance of frailty in patients with cardiovascular disease. Eur Heart $\mathrm{J}$. 2014;35(26):1726-31.(PMID: 24864078).

12. Kera $T$, Kawai $H$, Yoshida $H$, et al. Physical and psychological characteristics of the communitydwelling elderly with heart disease. Nihon Koshu
Eisei Zasshi. 2017;64(1):3-13. (PMID: 28228632).

13. Weinlein JC, Deaderick S, Murphy RF. Morbid obesity increases the risk for systemic complications in patients with femoral shaft fractures. J Orthop Trauma. 2015;29(3):91-5. (PMID: 24978946).

14. Murphy ME, McCutcheon BA, Kerezoudis $P$, et al. Morbid obesity increases risk of morbidity and reoperation in resection of benign cranial nerve neoplasms. Clin Neurol Neurosurg. 2016;148(1):1059. (PMID: 27434528).

15. Carron M, Zarantonello F, Leppariello G, Ori C. Obesity and perioperative noninvasive ventilation in bariatric surgery. Minerva Chirurgica. 2017;72 (3):24864. (PMID: 28482650).

16. Dixon $A E$, Peters $U$. The effect of obesity on lung function. Expert Rev Respir Med. 2018;12(9):755-67. (PMID: 30056777).

17. Lima $T R L$, Almeida VP, Ferreira $A S$, Guimarães FS, Lopes AJ. Handgrip strength and pulmonary disease in the elderly: What is the link? Aging Dis. 2019;10(5):1109-29. (PMID: 31595206).

18. Osman F, Franklyn JA, Daykin J, et al. Heart rate variability and turbulence in hyperthyroidism before, during, and after treatment. Am J Cardiol. 2004;94(4);465-9. (PMID: 15325930).

19. Pitzalis MV, Mastropasqua F, Massari F, et al. Assessment of cardiac vagal activity in patients with hyperthyroidism. Int J Cardiol. 1998;64 (2);145-51. (PMID: 9688433).

20. Eustatia-Rutten CFA, Corssmit EPM, Heemstra KA, et al. Autonomic nervous system function in chronic exogenous subclinical thyrotoxicosis and the effect of restoring euthyroidism. J Clin Endocrinol Metab. 2008;93(7);2835-41. (PMID: 18397977).

21. Pitzalis MV, Mastropasqua F, Massari F, et al. Assessment of cardiac vagal activity in patients with hyperthyroidism. Int J Cardiol. 1998;64(2):145-51. (PMID: 9688433).

22. Pasqualetti G, Tognini S, Polini A, Caraccio N, Monzani F. Is subclinical hypothyroidism cardiovascular risk factor in the elderly? Journal of Clinical Endocrinology and Metabolism. 2013;98(6);2256-66. (PMID: 23559085).

23. Weinberg $A D$, Brennan $M D$, Gorman $C A$, Marsh HM, O'fallon WM. Outcome of Anesthesia and Surgery in Hypothyroid Patients. Arch Intern Med. 1983;143(5):893-7. (PMID: 6679233). 
24. Giulea C, Enciu O, Toma EA, Martin S, Fica S, Miron A. Total thyroidectomy for malignancy - is central neck dissection a risk factor for recurrent nerve injury and postoperative hypocalcemia? A tertiary center experience in romania. Acta Endocrinol (Copenh). 2019;15(1):80-5. (PMID: 31149064).

25. Narayanan S, Arumugam D, Mennona S, Wang M, Davidov T, Trooskin SZ. An Evaluation of Postoperative Complications and Cost After Short-Stay Thyroid Operations. Ann Surg Oncol. 2016;23(5):1440-5. (PMID: 26628433). 